\title{
Lagrangian statistics in isotropic turbulent flows with deterministic and stochastic forcing schemes
}

\author{
Jin-Cai Chen · Guo-Dong Jin · Jian Zhang
}

Received: 26 August 2014 / Revised: 10 October 2014 / Accepted: 29 October 2014

(C)The Chinese Society of Theoretical and Applied Mechanics and Springer-Verlag Berlin Heidelberg 2015

\begin{abstract}
Artificial input of energy into the flow is necessary to create and maintain a statistically stationary isotropic turbulence for sampling in studying the statistics. Due to the nonlinear coupling among different Fourier modes through the triadic interaction, whether or not various forcing schemes affect the statistics in turbulence is an important and open question. We present detailed comparison of Lagrangian statistics of fluids particles in forced isotropic turbulent flows in $128^{3}, 256^{3}$, and $512^{3}$ simulations, with Taylor-scale Reynolds numbers in the range of 64-171, using a deterministic and a stochastic forcing scheme, respectively. Several Lagrangian statistics are compared, such as velocity and acceleration autocorrelations, and moments of Lagrangian velocity increments. The differences in the Lagrangian statistics obtained from the two forcing schemes are shown to be small, indicating that the isotropic forcing schemes used have little effects on the Lagrangian statistics in the isotropic turbulence.
\end{abstract}

Keywords Isotropic turbulence - Deterministic forcing scheme Stochastic forcing scheme - Lagrangian statistical quantities

\section{Introduction}

Following fluid particles in turbulent flows from the Lagrangian viewpoint is conceptually natural and practically feasible to study turbulent mixing and dispersion of contam-

The project was supported by the 973 Program of China (2013CB834100), the National Natural Science Associate Foundation of China (U1230126), and the National Science Foundation of China (11472277 and 11232011).

J.-C. Chen · G.-D. Jin (四) · J. Zhang

LNM, Institute of Mechanics,

Chinese Academy of Sciences, 100190 Beijing, China

e-mail: gdjin@lnm.imech.ac.cn inants. Taylor [1] studied the single particle turbulent dispersion, Richardson [2] studied the relative turbulent dispersion of particle pairs. Batchelor $[3,4]$ built connection between the concentration field of contaminants and the statistics of fluid particle motions. Durbin [5] established a stochastic model of two-particle dispersion which is consistent with Richardson's 4/3 law. He and his coworkers developed a series of elliptic models for two-point, two-time correlation of Eulerian [6, 7] and Lagrangian [8] velocities, respectively, which have been powerful tools for turbulence measure [9]. In recent years, direct numerical simulation (DNS) and largeeddy simulation (LES) have been taken as main tools to explore the fundamental features of turbulence and its interaction with other physical process [10-23]. In order to obtain turbulence statistics under stationary conditions in isotropic turbulent flows, energy is fed into the flow at large scales to balance the energy dissipation at small scales by molecular viscosity in DNS or by both molecular viscosity and subgrid scale (SGS) eddy viscosity in LES. Otherwise, the unsteady or decaying turbulent flows complicate the analysis of the statistical quantities.

Different kinds forcing schemes are developed to feed energy into the flow to maintain statistically stationary conditions, see Ref. [24] and the references therein. In a deterministic forcing scheme [25], the energy in each of the first two wavenumber shells $(0.5<k \leqslant 1.5$ and $1.5<k \leqslant 2.5)$ is maintained constant and the ratio of the energy between the two shell is consistent with the Kolmogorov $k^{-5 / 3}$ scaling law. The stochastic forcing scheme developed by Eswaran and Pope [26] has been widely used in maintaining a stationary isotropic turbulence. It is an Uhlenbeck-Ornstein random process at each of the forced modes.

The forcing schemes are based on Kolmogorov's hypothesis [27] of statistical independence between large flow structure and small-scale structure at high Reynolds number. However, the nonlinear coupling among different modes in spectral space implies that the forced large-scale mode 
may affect the small-scale mode through the non-local triadic interaction involving one mode located in the forced lowwave number range and two modes located in high wavenumbers, so it is necessary to justify the validity of the use of low-wavenumber forcing in DNS. Eswaran and Pope [26] focused on the effect of forcing parameters with stochastic forcing scheme, which showed that small scale structures of the velocity field are insensitive to the details of forcing. Yeung and Pope [28] presented Eulerian energy and dissipation spectral functions in isotropic turbulence with stochastic scheme. They proved that large scales are much sensitive than the small scales to the forcing parameters. Overholt and Pope [29] proposed a deterministic forcing scheme and make a comparison with stochastic scheme, yielding similar results. Rosa et al. [25] employed both stochastic and deterministic forcing schemes to test the effects of large-scale driving schemes on the collision-related statistics such as radial distribution function and radial relative velocity. They found that the collision statistics were insensitive to the nature of forcing for small inertial particles. Since the relative dispersion of fluid particles is mainly determined by smallscale motions [30], the objective of the present study is to check whether the Lagrangian statistics are affected if different forcing schemes are adopted.

This paper is organized as follows. In Sect. 2, we describe the numerical methods for both flow field and fluid particles. In Sect. 3, we firstly validate the codes by comparing our results with those from the reference with the same forcing scheme. We then study the effects of forcing time scale in stochastic forcing scheme and finally compare the Lagrangian statistics between the results obtained from the deterministic scheme and those obtained using the stochastic scheme. We give the conclusions in Sect. 4.

\section{Numerical simulations}

The governing equations for turbulent flow and fluid particles motions are briefly described in this section.

\subsection{Flow field}

In spectral space, the Navier-Stokes equations for a forced isotropic and incompressible turbulent flow can be represented as

$\left(\frac{\partial}{\partial t}+v k^{2}\right) \hat{\boldsymbol{u}}(\boldsymbol{k}, t)=\boldsymbol{P}(\boldsymbol{k}) F(\boldsymbol{u} \times \boldsymbol{\omega})+\hat{\boldsymbol{f}}(\boldsymbol{k}, t)$,

where $\hat{\boldsymbol{u}}(\boldsymbol{k}, t)$ is a Fourier mode or the velocity in Fourier space, $\boldsymbol{k}=\left(k_{x}, k_{y}, k_{z}\right)$ the wavenumber vector and $k=|\boldsymbol{k}|$, $\boldsymbol{u}$ and $\boldsymbol{\omega}$ fluid velocity and vorticity in physical space, $v$ fluid kinematical viscosity. The projection tensor $P_{j m}=$ $\delta_{j m}-k_{j} k_{m} / k^{2}(j, m=1,2,3)$ and $F$ denotes a Fourier transform. The random artificial force $\hat{f}(k, t)$ is used to drive and maintain the turbulent flow at low wavenumbers.

In this paper, two schemes are used for the random forcing, one is the deterministic scheme and the other is the stochastic one. In the deterministic scheme, we keep the total energy in each of the first two wave number shells $(0.5<|\boldsymbol{k}|<1.5$ and $1.5<|\boldsymbol{k}|<2.5)$ constant in time and fix them at $E(1)=\alpha$ and $E(2)=\beta$, respectively, to satisfy the $k^{-5 / 3}$ law by rescaling the Fourier modes in the first two wave numbers. The rescaling is equivalent to a force term as follows: we discretize the Navier-Stokes equations into two sub-steps in time

$\hat{\boldsymbol{u}}^{*}=\hat{\boldsymbol{u}}^{(n)}+\left[\boldsymbol{P}(\boldsymbol{k}) F(\boldsymbol{u} \times \boldsymbol{\omega})-v k^{2} \hat{\boldsymbol{u}}^{(n)}\right] \mathrm{d} t$,

$\hat{\boldsymbol{u}}^{(n+1)}=\hat{\boldsymbol{u}}^{*}+\boldsymbol{f}(\boldsymbol{k})^{(n)} \mathrm{d} t$,

where in the wavenumber range $0.5 \leqslant k \leqslant 1.5$

$\boldsymbol{f}^{(n)}(\boldsymbol{k})=\frac{1}{\mathrm{~d} t}\left(\sqrt{\frac{\alpha}{\sum_{0.5 \leqslant k \leqslant 1.5} 0.5\left|\hat{\boldsymbol{u}}^{*}(\boldsymbol{k})\right|^{2}}}-1\right) \hat{\boldsymbol{u}}^{*}(\boldsymbol{k})$,

and in the wavenumber range $1.5 \leqslant k \leqslant 2.5$

$\boldsymbol{f}^{(n)}(\boldsymbol{k})=\frac{1}{\mathrm{~d} t}\left(\sqrt{\frac{\beta}{\sum_{1.5 \leqslant k \leqslant 2.5} 0.5\left|\hat{\boldsymbol{u}}^{*}(\boldsymbol{k})\right|^{2}}}-1\right) \hat{\boldsymbol{u}}^{*}(\boldsymbol{k})$.

We set $\alpha=0.55544$ and $\beta=0.159843$ in this paper [25]. In the wavenumber range $k>2.5, \boldsymbol{f}^{(n)}(\boldsymbol{k})=\mathbf{0}$.

In the stochastic forcing scheme [26,31], random acceleration is added to each component of velocity in the Fourier space modes with $|\boldsymbol{k}|<\sqrt{8}$. The force is a vectorvalued Uhlenbeck-Ornstein stochastic process. It is characterized by two parameters, namely, an acceleration variance $\sigma_{\mathrm{f}}^{2}$ and a forcing time scale $t_{\mathrm{f}}\left(\sigma_{\mathrm{f}}^{2}=447.3, t_{\mathrm{f}}=0.038\right.$ in this paper). The force is determined by

$\mathrm{d} \hat{b}_{j}=-\frac{\hat{b}_{j}}{t_{\mathrm{f}}} \mathrm{d} t+\sqrt{\left(\frac{\sigma_{\mathrm{f}}^{2}}{t_{\mathrm{f}}}\right)} \mathrm{d} W$,

where $\mathrm{d} W$ denotes a random normal distribution $(\langle\mathrm{d} W\rangle=0$, $\left.\left\langle\mathrm{d} W^{2}=\mathrm{d} t\right\rangle\right)$, and the Fourier mode of the forcing $\hat{f}_{i}$ can be determined by the projection of $\hat{b}$ onto the plane normal to wavenumber $\boldsymbol{k}$ so as to satisfy the divergence-free condition to avoid the effects of forcing on the pressure of the flow field

$\hat{f_{i}}=\hat{b}_{j} P_{i j}$.

The spatial resolution of the simulation was monitored by $k_{\max } \eta$. All of our simulations have $k_{\max } \eta$ values of approximately 1.5 to ensure the accuracy of higher-order quantities such as dissipation and derivative statistics [28].

\subsection{Particle motion}

Fluid particles tracking starts from the moment $t_{0}$ when the flow becomes statistically stationary. Fluid particles are initially seeded into the flow domain at random locations $\boldsymbol{x}_{0}$.

Each particle is advected by the fluid flow

$\frac{\mathrm{d} \boldsymbol{x}^{+}\left(t ; \boldsymbol{x}_{0}, t_{0}\right)}{\mathrm{d} t}=\boldsymbol{u}^{+}\left(t, \boldsymbol{x}^{+} ; \boldsymbol{x}_{0}, t_{0}\right)$ 
where $\boldsymbol{x}^{+}\left(t ; \boldsymbol{x}_{0}, t_{0}\right)$ and $\boldsymbol{u}^{+}\left(t, \boldsymbol{x}^{+} ; \boldsymbol{x}_{0}, t_{0}\right)$ are the position and velocity of the tracers at time $t$.

The Lagrangian velocity $\boldsymbol{u}^{+}(\boldsymbol{x}, t)$ is obtained from the Eulerian velocity field $\boldsymbol{u}(\boldsymbol{x}, t)$ by a six-point Lagrangian interpolation in each spatial direction. Once the velocity $\boldsymbol{u}^{+}(\boldsymbol{x}, t)$ is computed, we used a fourth-order AdamsBashforth method [32] to calculate particle displacements.

\section{Results and discussion}

3.1 Statistics of the flow fields under different forcing schemes

Table 1 lists some key parameters of the flow: the kinematic viscosity $v$, time step $\delta t$, Kolmogorov length $\eta$, Kolmogorov time $\tau_{\eta}$, transverse Taylor microscale $\lambda$, integral length scale $L_{\mathrm{s}}$, large-eddy turnover time $T_{\mathrm{e}}$, integral length scale of the longitudinal spatial velocity correlation $L_{\mathrm{f}}$, fluctuating velocity $u^{\prime}$, energy dissipation rate $\epsilon$, Taylor-microscale Reynolds number $R_{\lambda}$, spatial resolution parameter $k_{\max } \eta$, CFL number, skewness $S$ and the flatness $F$ of the velocity gradient.

Figure 1 plots the comparison between the compensated energy spectra of the isotropic turbulent flow fields in the $512^{3}$ grids generated from the deterministic forcing scheme and stochastic forcing scheme, respectively. We can observe that the two spectra overlap at most of the normalized wavenumbers, and there is a better resolution of the dissipative scale in the flow generated from the deterministic forcing scheme. The Kolmogorov constant 1.62 is plotted for comparison in the inertial range.

Table 1 Parameters and statistics of the simulated flows using the stochastic and deterministic forcing schemes

\begin{tabular}{|c|c|c|c|c|}
\hline & \multicolumn{2}{|c|}{ Stochastic forcing scheme } & \multicolumn{2}{|c|}{ Deterministic forcing scheme } \\
\hline & $256^{3}$ & $512^{3}$ & $256^{3}$ & $512^{3}$ \\
\hline$v$ & $4.5 \times 10^{-2}$ & $2.0 \times 10^{-2}$ & $3.5 \times 10^{-3}$ & $1.4 \times 10^{-3}$ \\
\hline$\delta t$ & $3.0 \times 10^{-5}$ & $2.3 \times 10^{-5}$ & $1.0 \times 10^{-3}$ & $4.0 \times 10^{-4}$ \\
\hline$\eta$ & $1.171 \times 10^{-2}$ & $6.850 \times 10^{-3}$ & $2.132 \times 10^{-2}$ & $1.072 \times 10^{-2}$ \\
\hline$\tau_{\eta}$ & $3.430 \times 10^{-3}$ & $2.340 \times 10^{-3}$ & $1.299 \times 10^{-1}$ & $8.210 \times 10^{-2}$ \\
\hline$\lambda$ & $2.554 \times 10^{-1}$ & $1.677 \times 10^{-1}$ & $4.352 \times 10^{-1}$ & $2.758 \times 10^{-1}$ \\
\hline$L_{\mathrm{s}}$ & 1.002 & 1.047 & 1.502 & 1.469 \\
\hline$T_{\mathrm{e}}$ & $9.672 \times 10^{-2}$ & $9.384 \times 10^{-2}$ & 3.618 & 3.632 \\
\hline$L_{\mathrm{f}}$ & 1.895 & 1.882 & 3.132 & 3.150 \\
\hline$u^{\prime}$ & 19.59 & 20.06 & 0.866 & 0.868 \\
\hline$\epsilon$ & 3980 & 4309 & 0.208 & 0.210 \\
\hline$R_{\lambda}$ & 111.20 & 168.15 & 107.76 & 171.02 \\
\hline$k_{\max } \eta$ & 1.556 & 1.670 & 2.697 & 2.727 \\
\hline$C F L$ & 0.17 & 0.26 & 0.21 & 0.20 \\
\hline$S$ & -0.521 & -0.533 & -0.484 & -0.528 \\
\hline$F$ & 5.493 & 5.940 & 5.163 & 6.162 \\
\hline
\end{tabular}

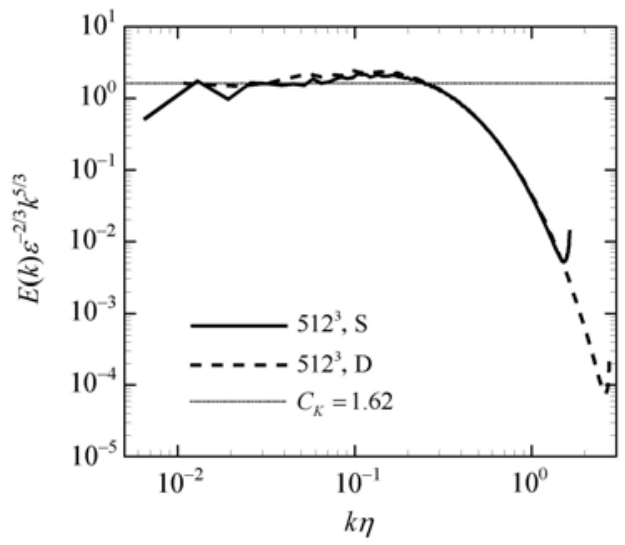

Fig. 1 Energy spectra of turbulent flow fields generated by the deterministic and stochastic forcing scheme, where " $D$ " in the legends denotes the deterministic scheme and " $\mathrm{S}$ " the stochastic forcing scheme

3.2 Validation of the Lagrangian statistics against published results

In order to validate our codes for capturing the Lagrangian statistics, we choose several quantities, including the Lagrangian velocity and acceleration autocorrelation functions, moments of Lagrangian velocity increments, to make comparisons with the modeling results of Yeung and Pope [28] in which the same stochastic forcing scheme was used. The Lagrangian velocity autocorrelation function is defined as

$\rho_{\mathrm{L}}(\tau)=\frac{\left\langle u^{+}(t) u^{+}(t+\tau)\right\rangle}{\left\langle u^{+}(t) u^{+}(t)\right\rangle}$,

where $u^{+}$denotes a component of Lagrangian velocity $\boldsymbol{u}^{+}$. The velocity increment over a time interval $\tau$ is defined as $\Delta_{\tau} u^{+}(t)=u^{+}(t+\tau)-u^{+}(t)$, its $m$-th-order moment, namely, the $m$-th-order Lagrangian velocity structure function, is denoted using $D_{m}^{\mathrm{L}}(\tau)=\Delta_{\tau}^{m} u^{+}(t)$. The flatness factor is $\mu_{4}(\tau) \equiv D_{4}^{\mathrm{L}}(\tau) / D_{2}^{\mathrm{L}}(\tau)^{2}$, and the acceleration is $\boldsymbol{a}^{+}(t+h / 2)=$ $(1 / h) \Delta_{h} \boldsymbol{u}^{+}(t)$, where $h$ is a time interval.

Figure 2a presents the comparison of Lagrangian velocity autocorrelation functions obtained from our DNS simulations and the results from Ref. [28]. It shows a good agreement against the normalized time lag with the Lagrangian integral timescale $T_{\mathrm{L}}=\int_{0}^{\infty} \rho_{\mathrm{L}}(\tau) \mathrm{d} \tau$. Figure $2 \mathrm{~b}$ presents the acceleration autocorrelation functions against the normalized time lag $\tau / \tau_{\eta}$ for different Reynolds numbers. The lines denote the data from Ref. [28]. All the cases overlap well: both of the case with $R_{\lambda}=64$ and the case with $R_{\lambda}=84$ are quite similar to those from Ref. [28] with $R_{\lambda}=63$ and $R_{\lambda}=90$, respectively. Figure $2 \mathrm{c}$ presents the second-order structure functions $D_{2}^{\mathrm{L}}(\tau)$ against the normalized time scale $\tau / \tau_{\eta}$. We compare the data of $128^{3}$ calculation at $R_{\lambda}=64$, and 90 from Ref. [28] with our results. The agreements of the cases with $R_{\lambda}=64$ and $R_{\lambda}=84$ suggest that the second-order statistics in our simulations are sufficiently accurate. Figure $2 \mathrm{~d}$ shows the flatness factors of the velocity-increment against the normalized time lag $\tau / \tau_{\eta}$, which is the normalized fourth-order 

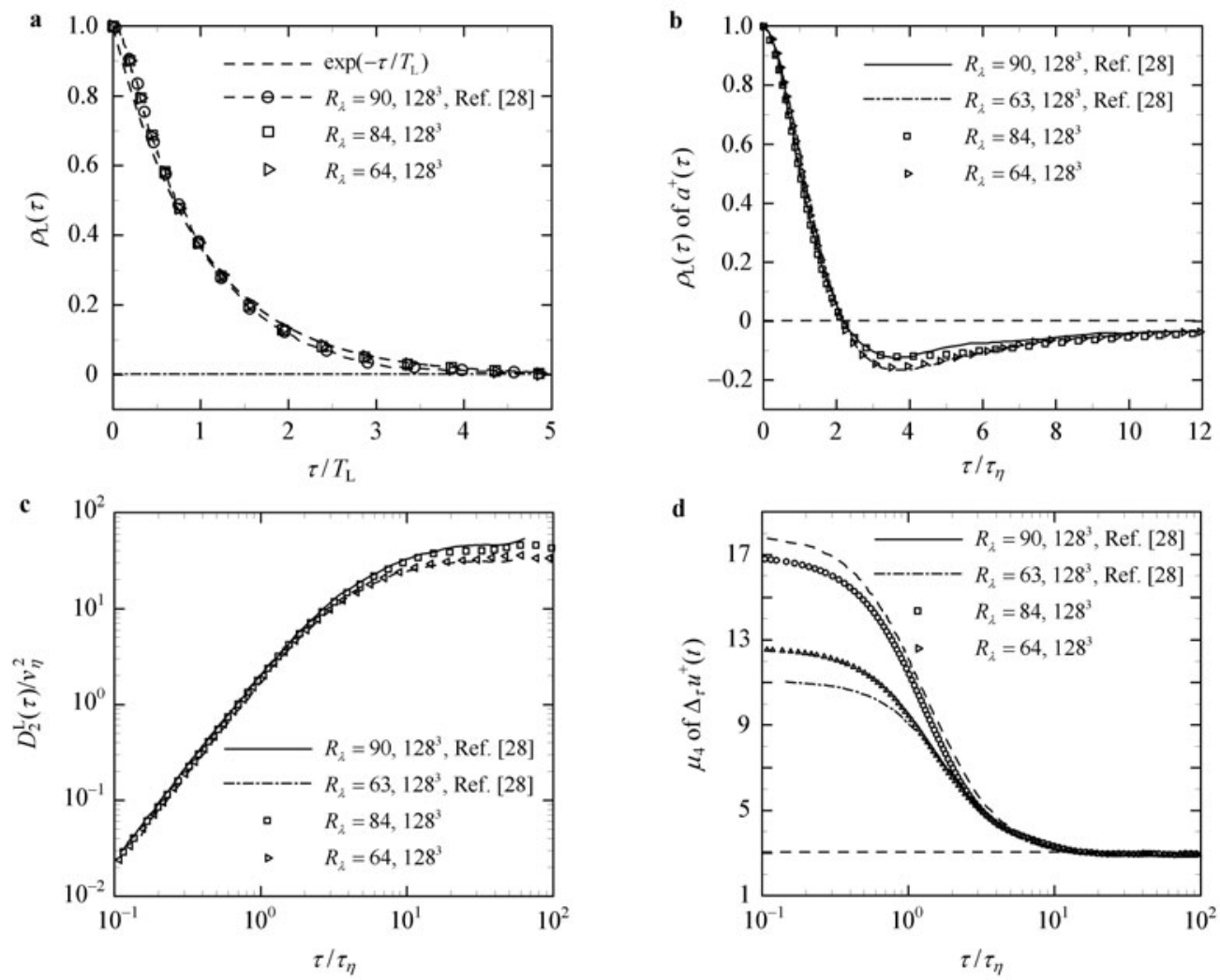

Fig. 2 Comparison of Lagrangian statistics from the $128^{3}$ simulations using the stochastic forcing scheme with the results from Ref. [28]. a Lagrangian velocity autocorrelation functions $\rho_{\mathrm{L}}(\tau)$ against the normalized time lag $\tau / T_{\mathrm{L}}$; $\mathbf{b}$ Autocorrelation functions of acceleration components against the normalized time lag $\tau / \tau_{\eta} ; \mathbf{c}$ The second-order Lagrangian velocity structure functions $D_{2}^{\mathrm{L}}(\tau)$ against $\tau / \tau_{\eta} ; \mathbf{d}$ Flatness factors of velocity increments against $\tau / \tau_{\eta}$. The horizontal dashed line denotes the value of a Gaussian distribution

moment providing a quantitative measure apart from being Gaussian. At small time lags, the values of the flatness factors, which increase with the Reynolds number, are much higher than the Gaussian value 3. As can be seen from the curves, our result are in agreement with the results from Ref. [28] at similar Reynolds numbers except at a very short time lags $\left(\tau<\tau_{\eta}\right)$. All of the above comparisons indicate that our simulation can capture accurate results for Lagrangian statistics.

\subsection{Effects of the time scale $t_{\mathrm{f}}$ in stochastic forcing scheme}

In the former subsection we have introduced two forcing schemes, namely, the deterministic and stochastic forcing scheme. The deterministic scheme injects energy into the flow depending on a given energy spectrum, while the stochastic scheme involves two parameters, the time scale $t_{\mathrm{f}}$ and the variation $\sigma_{\mathrm{f}}^{2}$. Thus, in order to study the effects of $t_{\mathrm{f}}$ and $\sigma_{\mathrm{f}}^{2}$ on Lagrangian statistics, we performed four simulations with different parameters $\left(t_{\mathrm{f}}=0.014,0.026,0.038\right.$, 0.050).

In the stochastic forcing scheme, the average dissipation rate (which is also the average rate of energy input) could be expressed as [26]

$\epsilon=4 N_{\mathrm{f}} \sigma_{\mathrm{f}}^{2} t_{\mathrm{f}} \times \frac{1}{1+t_{\mathrm{f}}\left(\sigma_{\mathrm{f}}^{2} t_{\mathrm{f}} N_{\mathrm{f}} k_{0}^{2}\right)^{1 / 3} / \beta}$, where the total number of forcing modes $N_{\mathrm{f}}=80$, the lowest wavenumber $k_{0}=1$ and the fitting coefficient $\beta=0.8$. Since the term $t_{\mathrm{f}}\left(\sigma_{\mathrm{f}}^{2} t_{\mathrm{f}} N_{\mathrm{f}} k_{0}^{2}\right)^{1 / 3}$ roughly represents the ratio of the forcing time scale to the time scale of large-scale flow and $t_{\mathrm{f}}$ must be much smaller than the large eddy turnover time [33], we set the term $\sigma_{\mathrm{f}}^{2} t_{\mathrm{f}}$ to be a constant so that the Reynolds numbers could be kept approximately equivalent for all the simulations. Several key parameters are listed in Table 2, such as the forcing time scale $t_{\mathrm{f}}$, acceleration variance $\sigma_{\mathrm{f}}^{2}$, Kolmogorov length scale $\eta$ and time scale $\tau_{\eta}$ and Taylor-microscale Reynolds number $R_{\lambda}$.

Figures $3 a-3 c$ represent the Lagrangian velocity, acceleration autocorrelation functions and the second-order Lagrangian velocity structure functions from the $256^{3}$ simula-

Table 2 Parameters and statistics of the simulated flows for different forcing time scale $t_{\mathrm{f}}$

\begin{tabular}{llllll}
\hline$t_{\mathrm{f}}$ & $\sigma_{\mathrm{f}}^{2}$ & $\eta$ & $\tau_{\eta}$ & $T_{\mathrm{L}}$ & $R_{\lambda}$ \\
\hline 0.014 & 1214.1 & 0.01184 & 0.00312 & 0.03582 & 115 \\
0.026 & 653.7 & 0.01221 & 0.00331 & 0.03600 & 111 \\
0.038 & 447.3 & 0.01236 & 0.00340 & 0.04015 & 111 \\
0.050 & 339.9 & 0.01299 & 0.00375 & 0.04045 & 106 \\
\hline
\end{tabular}



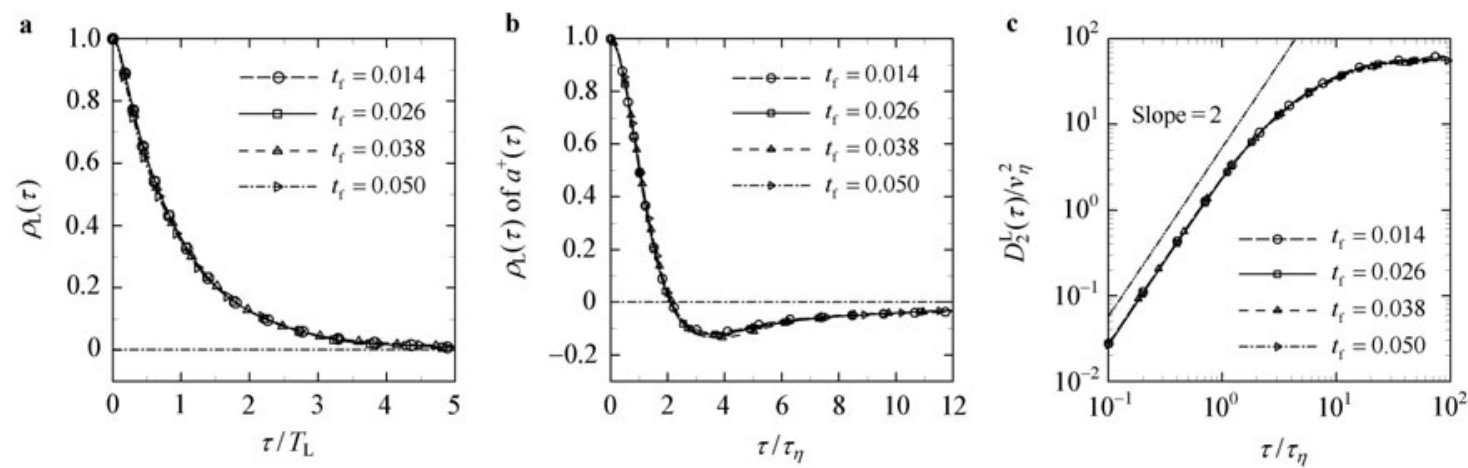

Fig. 3 Effect of forcing time scale on Lagrangian statistics. a Lagrangian velocity autocorrelation functions $\rho_{\mathrm{L}}(\tau)$ against the normalized time lag $\tau / T_{\mathrm{L}}$; b Autocorrelation functions of acceleration components against the normalized time lag $\tau / \tau_{\eta}$; $\mathbf{c}$ The second-order Lagrangian velocity structure functions $D_{2}^{\mathrm{L}}(\tau)$ against $\tau / \tau_{\eta}$. From $256^{3}$ simulations, $R_{\lambda} \approx 111$

tions, respectively. The curves corresponding to each time scale demonstrate a remarkable consistency in these plots, which implies that these statistics are insensitive to the forcing time scale. As the acceleration variance $\sigma_{\mathrm{f}}^{2}$ changes with the time scale $t_{\mathrm{f}}$, the results outlined above seem to show a fact that, for a given Reynolds number and forcing modes in stochastic forcing scheme, the values of small scale statistics can be expected to be independent of the choice of forcing time scale, while the large scale statistics show a slightly dependence on $t_{\mathrm{f}}$ in terms of the integral time scale $T_{\mathrm{L}}$.

3.4 Lagrangian statistics from the deterministic and stochastic forcing schemes

In this section, we report the DNS results of Lagrangian statistics obtained from the $256^{3}$ and $512^{3}$ simulations at several Reynolds numbers. We focus on the effects of two types of forcing schemes, i.e., the deterministic and stochastic ones on the Lagrangian statistical quantities, such as the Lagrangian velocity autocorrelation functions, acceleration autocorrelation functions, Lagrangian velocity structure function and its local scaling exponents.

Firstly, we present the Lagrangian velocity autocorrelations, $\rho_{\mathrm{L}}(\tau)$, which is important for single-particle turbulent diffusion. Figure 4 shows the Lagrangian velocity autocorrelation functions against time lag normalized by the Lagrangian integral timescale $T_{\mathrm{L}}, \tau / T_{\mathrm{L}}$. By comparing the cases at similar Reynolds number obtained from different forcing schemes, we find that all the curves overlap together, which indicates that $T_{\mathrm{L}}$ is a proper timescale to characterize the Lagrangian velocity autocorrelations and the forcing scheme has little effects on the normalized correlation functions.

We now consider the autocorrelation function of acceleration, $\rho_{a}(\tau)$. Figure 5 shows the $256^{3}$ and $512^{3}$ simulation results against the normalized time lag $\tau / \tau_{\eta}$. The autocorrelation functions of acceleration take negative values at large time lags and have little gaps on the zero-crossing time for different Reynolds number $R_{\lambda}$ when normalized by $\tau_{\eta}$. The rapid decorrelation of Lagrangian acceleration implies that the correlation of Lagrangian acceleration is a short timescale process and the overlaps of the curve obtained from different forcing scheme indicates that the forcing schemes have little effects on small-scale processes.

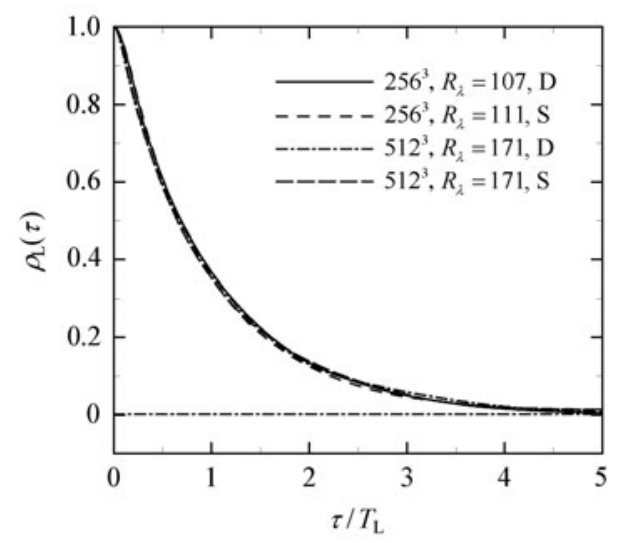

Fig. 4 Lagrangian velocity autocorrelation functions $\rho_{\mathrm{L}}(\tau)$ against time lag normalized by the Lagrangian integral timescale $T_{\mathrm{L}}, \tau / T_{\mathrm{L}}$, where " $\mathrm{D}$ " denotes the cases for deterministic forcing, and " $\mathrm{S}$ " for stochastic forcing

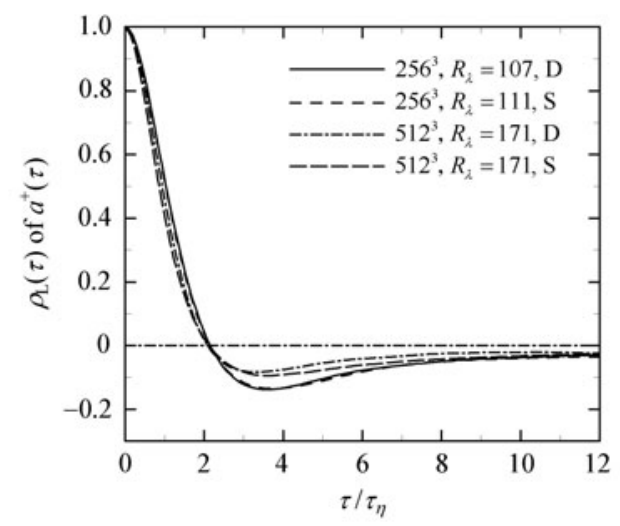

Fig. 5 Autocorrelation functions of acceleration components as a function of time lag normalized by the Kolmogorov scale simulated for different forcing schemes ("S": stochastic, "D": deterministic). The autocorrelations are nearly zero for time lags beyond the range 
shown

In the following, we shall analyze the statistical property of the Lagrangian velocity increments. The secondorder Lagrangian structure function $D_{2}^{\mathrm{L}}(\tau)$ is defined as the variance of the velocity increment. For very small $\tau, D_{2}^{\mathrm{L}}(\tau)$ is in proportion to $\tau^{2}$. Thus we have

$\frac{D_{2}^{\mathrm{L}}(\tau)}{v_{\eta}^{2}}=a_{0}\left(\tau / \tau_{\eta}\right)^{2}$,

where $a_{0}$ is a universal constant and $v_{\eta}$ is the kolmogorov velocity scale. The second-order structure functions are shown in Fig. 6 by using a log-log plot where we can observed a line with the slope of 2 for small $\tau<2 \tau_{\eta}$, in agreement with Ref. [28]. The constant $a_{0}$ is found to vary with Reynolds number.

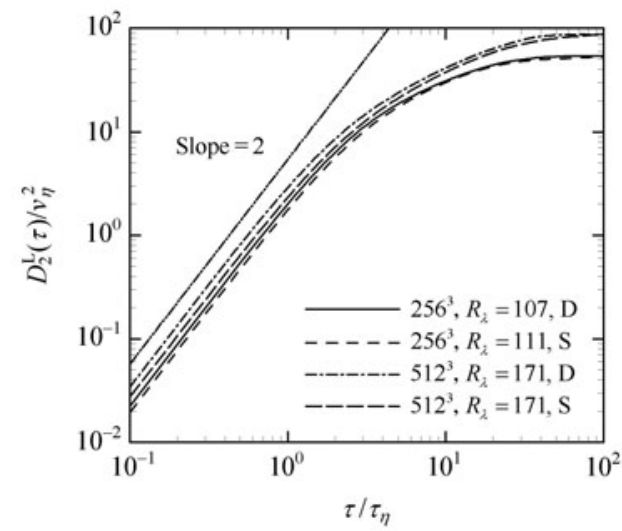

Fig. 6 The second-order Lagrangian velocity structure functions $D_{2}^{\mathrm{L}}(\tau)$ against normalized time $\tau / \tau_{\eta}$, "D" and "S" denote the deterministic and stochastic forcing schemes, respectively

For large $\tau / \tau_{\eta}$, the normalized second-order structure function $D_{2}^{\mathrm{L}}(\tau) / v_{\eta}^{2}$ is observed to take value of $2 u^{\prime 2} / v_{\eta}^{2}$, increasing with $R_{\lambda}$, as expected, where $u^{\prime}$ is the root mean square (rms) of the fluctuation velocity. It consists with the fact that the second-order structure function is twice the velocity variance at large times $\left(t \gg T_{\mathrm{L}}\right)$.

We shall now present the scaling exponents of highorder moments of the velocity increments. In the inertial range of an isotropic turbulent flow, $\tau_{\eta} \ll \tau \ll T_{\mathrm{L}}$, different moments of the structure functions could be expressed as $D_{p}^{\mathrm{L}}(\tau) \sim(\epsilon \tau)^{p / 2}$ if we do not consider the intermittence effect [34]. However, the tail of the probability density functions (p.d.f.) of $\Delta_{\tau} u^{+}(t)$ becomes non-Gaussian at small time lags, implying the existence of intermittency. It means that the exponents $\chi^{(p)}$ of the dimensional law $D_{p}^{\mathrm{L}}(\tau) \sim \tau^{\chi^{(p)}}$ is not equal to $p / 2$. In this work, we calculate the local scaling exponents relative to another moment versus the second order

$\chi^{(p)}(\tau) \equiv \frac{\mathrm{d} \log D_{p}^{\mathrm{L}}(\tau)}{\mathrm{d}} \log D_{2}^{\mathrm{L}}(\tau)$,

which are related, in presence of pure power-law scaling.
In Fig. 7 we show the local exponents for $p=4$, $6,8,10$. We can observe that the Lagrangian scaling reflects strong intermittency. All of our cases show underestimated value of the scaling exponents as expected, which is in agreement with the results of Ref. [34]. The structure function scaling exponents characterize the small scale properties of the turbulence. The deterministic and stochastic forcing schemes give the same results for the exponents of the structure function. Thus, we can know that different forcing schemes have little influence on the small-scale statistics.

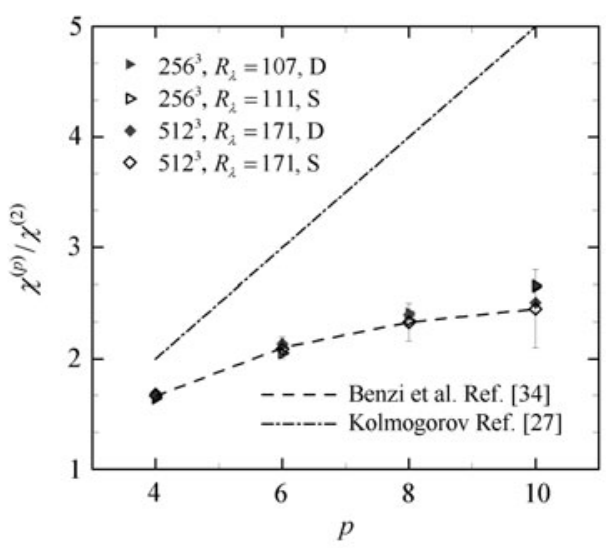

Fig. 7 Lagrangian local scaling exponents for hyper-structure functions, $\chi^{(p)} / \chi^{(2)}$ for $p=4,6,8,10$. Simulations with deterministic forcing are labeled as " $D$ " and those with stochastic forcing are labeled as "S". Dashed line and error bars represent DNS results at $R_{\lambda}=600$, obtained by Benzi et al. [34]

\section{Conclusions}

In conclusion, we study the Lagrangian statistical quantities through direct numerical simulations of a forced isotropic turbulence using the deterministic and the stochastic forcing schemes, respectively. The code was validated by comparing our results with those from Ref. [28] both using the stochastic forcing scheme. Small-scale Lagrangian statistics, such as acceleration autocorrelation function and structure function scaling exponents, were proved to be little affected by the forcing schemes used in the paper. Our results justify the use of forcing in Lagrangian turbulence, which also implies that it is possible to apply large-scale forcing to the study of small scale behavior in particle-laden turbulence and turbulent combustion.

Acknowledgements G. D. Jin/Jin benefitted from the hospitality of the Nordic Institute for Theoretical Physics under the auspices of the program "Dynamics of Particles in Flows: Fundamentals and Applications" in June 2014 in Sweden. We would like to thank the two anonymous reviewers for the constructive comments and suggestions.

\section{References}


1 Taylor, G.I.: Diffusion by continuous movements. Proc. Lond. Math. Soc. Ser. 220, 196-211 (1921)

2 Richardson, L.F.: Atmospheric diffusion shown on a distanceneighbor graph. Proc. Roy. Soc. London Ser. A 110, 709-737 (1926)

3 Batchelor, G.K.: Diffusion in a field of homogeneous turbulence. I. Eulerian analysis. Aust. J. Sci. Res. 2, 437-450 (1949)

4 Batchelor, G.K.: Diffusion in a field of homogeneous turbulence. II. The relative motion of particles. Proc. Camb. Phil. Soc. 48, 345-361 (1952)

5 Durbin, P.A.: A stochastic model of two-particle dispersion and concentration fluctuations in homogeneous turbulence. J. Fluid Mech. 100, 279-302 (1980)

6 He, G.W., Zhang, J.B.: Elliptic model for space-time correlations in turbulent shear flows. Phys. Rev. E 73, 055303(R) (2006)

7 Zhao, X., He, G.W.: Space-time correlations of fluctuating velocities in turbulent shear flows. Phys. Rev. E 79, 046316 (2009)

8 He, G.W., Jin, G.D., Zhao, X.: Scale-similarity model for Lagrangian velocity correlations in isotropic and stationary turbulence. Phys. Rev. E 80, 066313 (2009)

9 James, H., Guenter, A.: Reynolds-number measurements for low-Prandtl-number turbulent convection of large-aspect-ratio samples. J. Fluid Mech. 725, 664-680 (2013)

10 Moin, P., Mahesh, K.: Direct numnerical simulation: A tool in turbulence research. Annu. Rev. Fluid Mech. 30, 539-578 (1998)

11 Pope, S.B.: Turbulent Flows, Cambridge University Press \& Beijing World Publishing Corporation, Beijing (2000)

12 He, G.W., Rubinstein, R., Wang, L.P.: Effects of subgrid-scale modeling on time correlations in large eddy simulation. Phys. Fluids 14, 2186-2193 (2002)

13 Yeung, P.K.: Lagrangian investigation of turbulence. Annu. Rev. Fluid Mech. 34, 115-142 (2002)

$14 \mathrm{He}$, G.W., Wang, M., Lele, S.K.: On the computation of spacetime correlations by large-eddy simulation. Phys. Fluids 16, 3859-3867 (2004)

15 Yang, Y., He, G.W., Wang, L.P.: Effects of subgrid-scale modeling on Lagrangian statistics in large-eddy simulation. J. Turbu. 9, N8 (2008)

16 Fang, L., Shao, L., Bertoglio, J.P., et al.: An improved velocity increment model based on Kolmogorov equation of filtered velocity. Phys. Fluids 21, 065108 (2009)

17 Toschi, F., Bodenschatz, E.: Lagrangian properties of particles in turbulence. Annu. Rev. Fluid Mech. 41, 375-404 (2009)

18 Jin, G.D., He, G.W., Wang, L.P.: Large-eddy simulation of turbulent collision of heavy particles in isotropic turbulence. Phys. Fluids 22, 055106 (2010)

19 Ayala, O., Rosa, B., Wang, L.P., et al.: Effects of turbulence on the geometric collision rate of sedimenting droplets. Part
1. Results from direct numerical simulation. New J. Phys. 10, 075015 (2008)

20 Jin, G.D., He, G.W., Wang, L.P., et al.: Subgrid scale fluid velocity timescales seen by inertial particles in large-eddy simulation of particle-laden turbulence. Int. J. Multiphase Flow 36, 432-437 (2010)

21 Zamansky, R., Coletti, F., Massot, M., et al.: Radiation induces turbulence in particle-laden fluids. Phys. Fluids 26, 071701 (2014)

22 Jin, G.D., He, G.W.: A nonlinear model for the subgrid timescale experienced by heavy particles in large eddy simulation of isotropic turbulence with a stochastic differential equation. New J. Phy. 15, 035011 (2013)

23 Jin, G.D., Wang, Y., Zhang, J., et al.: Turbulent clustering of point particles and finite-size particles in isotropic turbulent flows. Ind. Eng. Chem. Res. 52, 11294-11301 (2013)

24 Rosales, C., Meneveau, C.: Linear forcing in numerical simulations of isotropic turbulence: Physical space implementations and convergence properties. Phys. Fluids 17, 095106 (1992)

25 Rosa, B., Parishani, H., Ayala, O., et al.: Kinematic and dynamic pair collision statistics of sedimenting inertial particles relevant to warm rain initiation. J. Phys.: Conf. Ser. 27, 19211936 (2011)

26 Eswaran, E., Pope, S.B.: An examination of forcing in direct numerical simulations of turbulence. Comput. Fluids 16, 257278 (1988)

27 Kolmogorov, A.N.: The local structure of turbulence in incompressible viscous fluid for very large Reynolds numbers. Proc. Royal Soc. London 434, 9-13 (1991)

28 Yeung, P.K., Pope, S.B.: Lagrangian statistics from direct numerical simulations of isotropic turbulence. J. Fluid Mech. 207, 531-586 (1989)

29 Overholt, M., Pope, S.B.: A deterministic forcing scheme for direct numerical simulations of turbulence. Computers and Fluids 27, 11-22 (1998)

30 Yeung, P.K., Brasseur, J.G.: The response of isotropic turbulence to isotropic and anisotropic forcing at large scales. Phys. Fluids A 3, 884-897 (1991)

31 Wang, L.P., Maxey, M.R.: Settling velocity and concentration distribution of heavy particles in homogeneous isotropic turbulence. J. Fluid Mech. 256, 27-68 (1993)

32 Press, W.H., Teukolsky, S.A., Vetterling, W.T., et al.: Numerical Recipes in Fortran: The Art of Scientific Computing. Cambridge University Press, Cambridge (1992)

33 Rosa, B., Parishani, H., Ayala, O., et al.: Kinematic and dynamic collision statistics of cloud droplets from high-resolution simulations. New J. Phy. 15, 045032 (2013)

34 Benzi, R., Biferale, L., Fisher, R., et al.: Inertial range Eulerian and Lagrangian statistics from numerical simulations of isotropic turbulence. J. Fluid Mech. 653, 221-244 (2010) 